\title{
Lead Site II
}

National Cancer Institute

\section{Source}

National Cancer Institute. Lead Site II. NCI Thesaurus. Code C90409.

A bipolar electrocardiog ram limb lead which records the voltage between the negative electrode on the right thoracic limb and the positive electrode on the left pelvic limb. 